\title{
Influence of a stereo surface on the perceived tilt of a monocular line
}

\author{
FULVIO DOMINI \\ Brown University, Providence, Rhode Island \\ and \\ MYRON L. BRAUNSTEIN \\ University of California, Irvine, California
}

\begin{abstract}
This study addressed the issue of how a stereo-specified surface influences the perceived twodimensional orientation of a monocularly viewed line. In a series of three experiments, it was found that, in general, when a monocular line was embedded in a disparity field specifying a planar surface slanted about the horizontal axis, the tilt of the line depended on the slant of the surface in which it was embedded and on whether the line was presented in the left or in the right visual field. These results, predicted by perspective geometry, are compatible with the hypothesis that the monocular line is perceived as part of the stereo surface. Moreover, it was found that timing is a crucial factor in determining the strength of the effect. The effect was at a maximum when the monocular line and the stereo surface were presented together, with no prior presentation of the surface. The influence of the stereo surface on the monocular line was substantially reduced, however, when the surface alone preceded the combined presentation of the line and the surface.
\end{abstract}

The role of stereopsis in human depth perception has interested vision scientists since the beginning of the 19th century (Wheatstone, 1838). Similar patterns on the two eyes, the elements of which are slightly displaced in the two images, give rise to a compelling impression of threedimensionality even if no structure is apparent in the two monocular images (Julesz, 1960).

Most of the effort in the study of stereopsis has been devoted to understanding the mechanisms that attribute a three-dimensional (3-D) interpretation to matchable features (see Howard \& Rogers, 1995, for a review). However, it has also been pointed out that monocular regions (i.e., regions that are seen by only one eye) play a fundamental role in the interpretation process of stereo images. The presence of a monocular region is caused in a natural viewing situation by the presence of a depth discontinuity. In fact, when an object occludes another object, a part of the occluded object is seen only by one eye. Several empirical investigations have therefore exploited the role of monocular regions in the perception of stereo occlusions (see Anderson \& Nakayama, 1994, for a review). Nakayama and Shimojo, in a series of papers, have addressed the question of how monocular elements are resolved by the perceptual system when they are located in the vicinity of binocular elements specifying a depth dis-

This research was supported by NEI Grant 1R01EY12437 and NSF Grants SBR 9511198 and DBI 9724595 . Correspondence concerning this article should be addressed to F. Domini, Department of Cognitive and Linguistic Sciences, Brown University, P. O. Box 1978, Providence, RI 02912 (e-mail: fulvio_domini@brownedu). continuity. In particular, they have found that monocular texture elements are necessary to create the impression of a sharp depth edge (Nakayama \& Shimojo, 1990). Moreover, they have established that the rivalry mechanism is coupled to the mechanism that interprets monocular occlusions: When the monocular regions are not compatible with an ecologically valid interpretation, they are suppressed by a rivalrous textured background (Shimojo \& Nakayama, 1990). In more recent work, Shimojo and Nakayama (1994) have investigated the perceived depth of a monocular element in the vicinity of an occluding edge. Their findings indicate that when the element is present in the interocularly unpaired zone of the stereo occlusion, its perceived depth is stable and corresponds to the depth of the occluded surface. On the other hand, when the monocular element is superimposed on an interocularly paired zone, its perceived depth fluctuates. These results, together with the previously mentioned results, suggest that the perceptual system's interpretation of an unpaired monocular zone is consistent with the geometrical interpretation of occlusions.

The present study asks the question of how monocular elements are interpreted by the visual system when they are embedded in a smooth disparity field. Let us consider Figure 1, in which a slanted random-dot planar surface is seen binocularly by a hypothetical observer. For each random dot on the surface (black dots in Figure 1), there is a corresponding left and right projection. For these dots, the matching problem can be solved, and a clear percept arises. On the other hand, a set of elements aligned to form a line segment is added only, say, to the right image (gray 


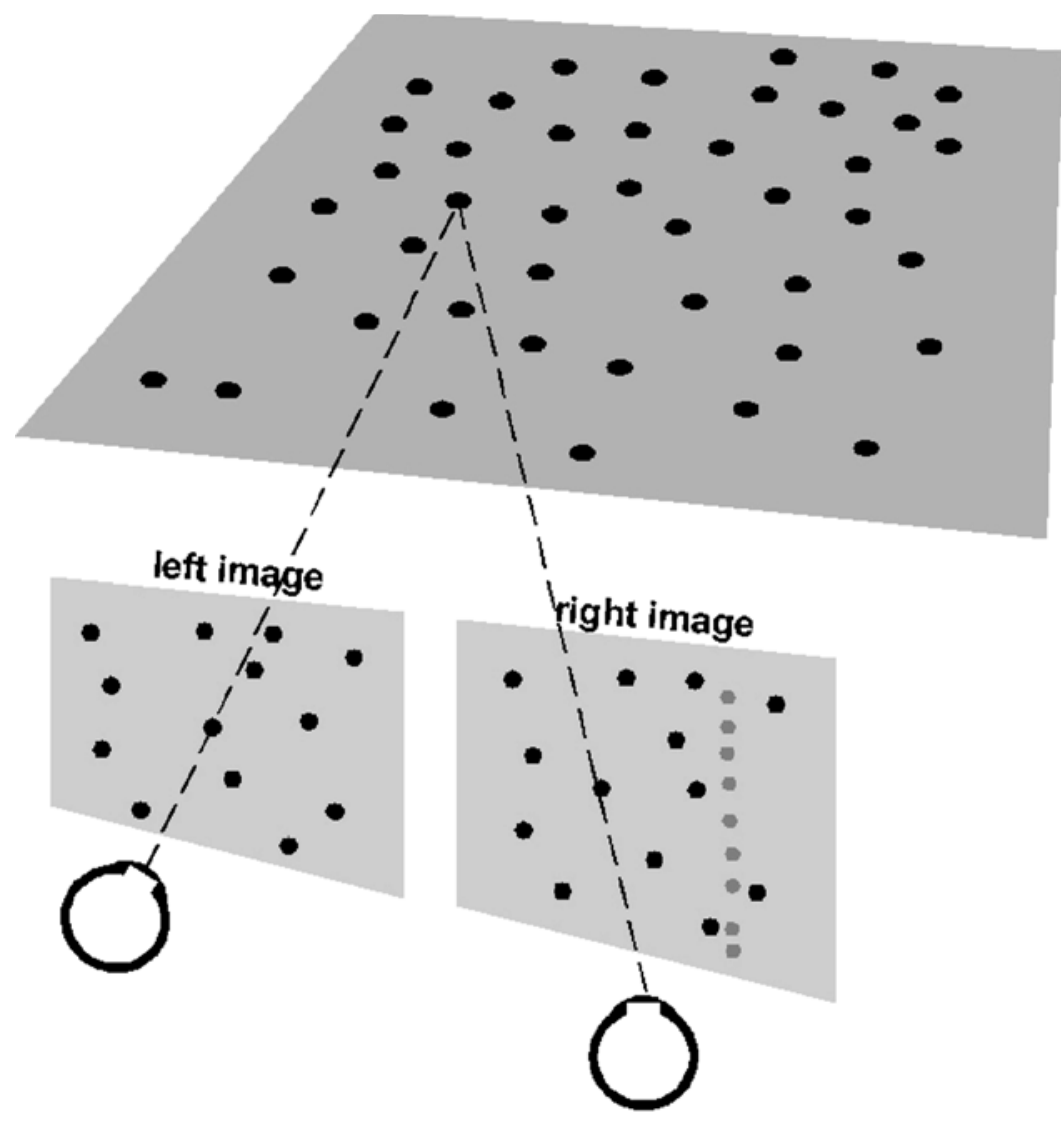

Figure 1. The viewing situation, showing a stereo-specif ied slanted planar surface with monocular elements forming a line added to the right eye image.

dots in Figure 1). These elements do not have any corresponding elements in the left image. How are these monocular elements treated by the visual system? The viewing situation sketched in Figure 1 does not allow any "ecologically valid" interpretation of the monocular line, since no occlusions are present that may determine a monocular region. However, the visual system must assign a 3-D orientation to the segment. A possible hypothesis is that the perceptual system "by default" locates the monocular segment on the stereo surface. ${ }^{1}$ As we will see, if the line is perceived as being part of the stereo surface, its perceived tilt, one of the two parameters that define the 3-D orientation of the line, should depend on the 3-D orientation of the stereo surface. We will therefore start by explaining how the 3-D orientation of a line can be defined.

The top panel of Figure 2 depicts a 3-D-oriented line. The two checkerboards, configured as a wall and as a floor, constitute a frame of reference. The slant of the line (Figure 2, bottom right panel) can be defined as the angle between the elevation plane that contains the line and the $x-y$ (the frontoparallel) plane. The tilt of the line (Figure 2, bottom left panel) is the angle between the (orthographic) projection of the line in the $x-y$ plane and the $x$-axis. If the hypothesis mentioned above is correct, we should ex- pect that the 3-D stereo surface influences the perceived tilt of the monocular line. This point can be understood by examining Figures 3 and 4. Figure 3 represents the cyclopean viewing of the 3-D surface and the monocular line segment depicted in Figure 1. The two lines that join the points $\mathrm{P}_{0}^{\prime}$ and $\mathrm{P}_{1}^{\prime}$ and the cyclopean viewpoint are the visual directions assigned to the two extremes of the line segment. If the monocular segment is perceived as belonging to the 3-D surface, its perceived tilt should depend on the slant of the 3-D surface. This fact is illustrated in Figure 4, where the three top panels represent the conditions in which the surface has a positive, a nil, and a negative slant. It can be observed that a "floor" surface should make the line appear to be tilted rightward, a frontoparallel surface should make the line appear vertical, and a "ceiling" surface should make the line appear to be tilted leftward.

It is very important to note that the three top panels represent the same perceived visual directions for the two extremes of the line segment, as can be observed by examining the three bottom panels of Figure 4 . The fact that the perceived tilt of the line is different in the three viewing situations depends only on the perceived relative position of $\mathrm{P}_{0}$ and $\mathrm{P}_{1}$. For example, if the bottom of the 

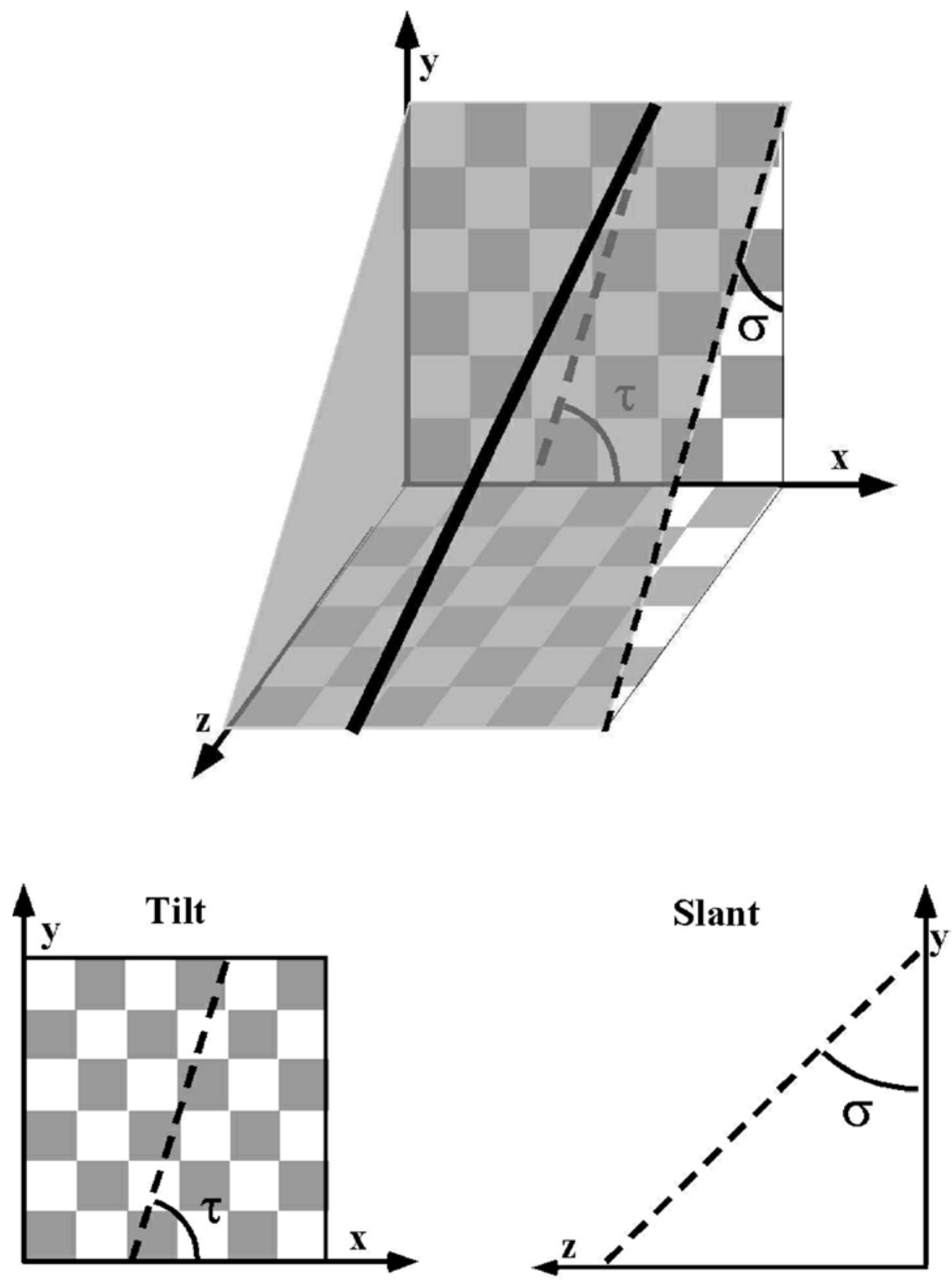

Figure 2. Description of the three-dimensional orientation of a line in terms of its slant $(\sigma)$ and tilt ( $\tau$; top panel). Tilt can be defined as the angle between the (orthographic) projection of the line on the $x-y$ plane and the $x$-axis (bottom left panel). Slant can be defined as the angle between the elevation plane that contains the line and the $x-y$ plane (bottom right panel).

segment $\left(\mathrm{P}_{0}\right)$ is perceived to be closer than the top of the segment $\left(\mathrm{P}_{1}\right)$, then $\mathrm{P}_{0}$ is perceived to be on the right of $\mathrm{P}_{1}$, and a rightward tilt should be perceived (left panels). Consequently, if the perceptual system interprets the vertical line in the monocular image as belonging to the slanted surface, the line should appear tilted, with the sign of its tilt dependent on the sign of the surface slant and the visual field in which the line is located. It can, in fact, be shown (see the Appendix) that the perceived horizontal separation between the two extremes of the line should have opposite signs, depending on whether the line is in the left or the right visual field. Figure 5 shows the predicted perceived orientations of a vertical monocular line in the left and right visual fields if the line is perceived to be located on a surface with positive slant. As can be inferred by looking at Figure 4, these predictions are opposite if the perceived depth separation has the opposite sign. ${ }^{2}$ 


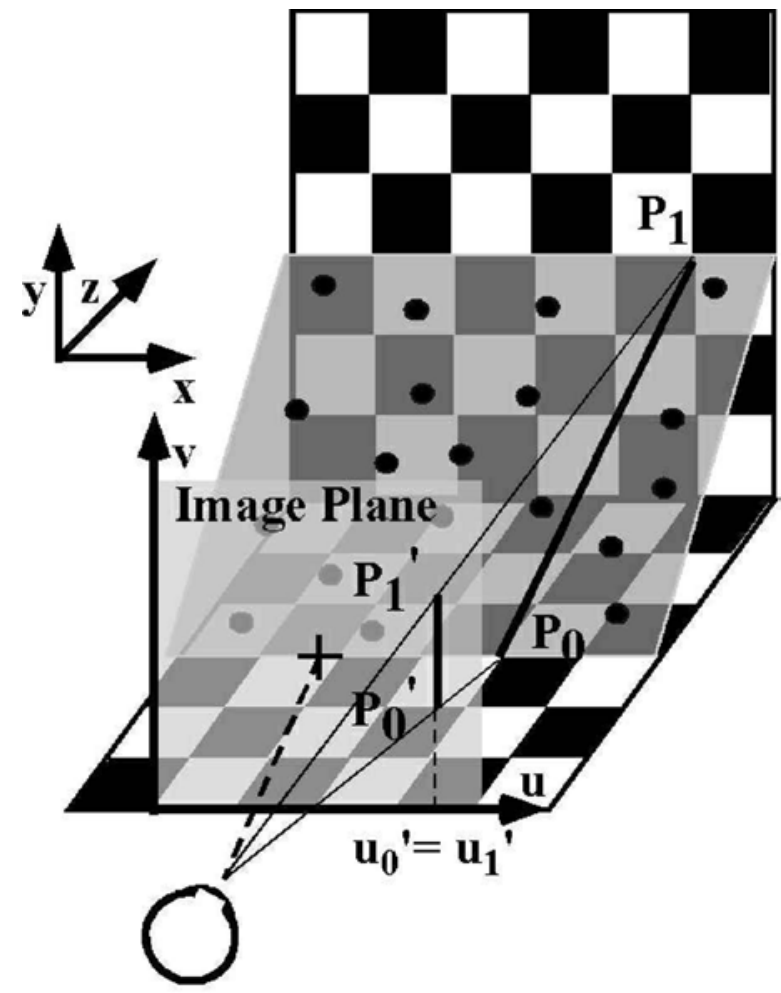

Figure 3. A three-dimensional (3-D) scene with a line depicted on a slanted random-dot surface. Although the line is tilted in 3-D space, it projects a vertical segment on the image plane.

These geometrical considerations show that the perceived tilt of a segment can vary even though the perceived visual directions of its extremes are constant. A constant perceived visual direction for the monocular line would be predicted by Hering's (1879/1942) law. In fact, according to Hering, the perceived visual direction of a monocular target depends only on the position of the target and is independent on the binocular elements that surround the target. However, recent findings indicate that the perceived visual direction of an unmatched element is influenced by nearby binocular regions (Erkelens, Muijs, \& van Ee, 1996; Erkelens \& van de Grind, 1994; Erkelens \& van Ee, 1997a, 1997b; Ono \& Mapp, 1995; Shimojo \& Nakayama, 1990; Shimono, Ono, Saida, \& Mapp, 1998). To make the qualitative predictions clear, we will assume that a unique visual direction is assigned to the two extremes of the line segment. We will then analyze whether our findings about perceived tilt of the monocular segment may have been produced by the influence of the binocular surfaces on the perceived visual direction of the segment extremes. Here, we anticipate that perceived visual direction cannot account for the results presented in this work, and we refer the reader to the end of the paper for a further discussion.

In summary, the main purpose of the experiments described in this paper is to test the hypothesis that the slant of stereo planar surface influences the perceived tilt of a monocular line segment as if the segment was perceived to be part of the planar surface. Moreover, we will also study the effect of timing of the stimulus events, since other studies of stereovision indicate that temporal manipulation is relevant. For example, timing has been found to be important in the perception of binocular rivalry (Wolfe, 1986) and in the depth contrast effect (Kumar \& Glaser, 1991, 1993; van Ee, Banks, \& Backus, 1999a).

\section{GENERAL METHOD}

\section{Observers}

Three naive observers at the University of California, Irvine participated in the first experiment. One of these observers and 3 additional naive observers participated in the third experiment. They were paid for their participation. Eight naive observers at Brown University participated in the second experiment. All the observers had normal or corrected-to-normal vision (20/40 on the Snellen eye chart).

\section{Apparatus}

The stimuli were presented on a high-resolution color monitor $(1,280 \times 1,024$ addressable locations $)$, under the control of a Silicon Graphics Indigo 2 workstation. The screen had a $120-\mathrm{Hz}$ refresh rate. The stimuli were presented stereoscopically, using LCD (liquid crystal) shuttered glasses that were synchronized with the monitor refresh rate. When, say, the right eye was occluded by the glasses and the left eye was not, the left image was presented on the screen. Therefore, the refresh rate of the stereo image was $60 \mathrm{~Hz}$. An anti-aliasing procedure was used: For point locations falling on a pixel boundary, the screen luminance was proportionally adjusted in the relevant addressable locations. The observer sat $32.5 \mathrm{~cm}$ from the screen. Head movement was restricted by use of a chinrest.

\section{Stimuli}

The basic stimulus used in the experiments consisted of 900 high-luminance random dots on a low-luminance background. The disparity field specified a planar surface slanted $-33^{\circ}$ (ceiling), $0^{\circ}$ (frontoparallel plane), or $+33^{\circ}$ (floor) about the horizontal axis. The dots were randomly distributed, with uniform probability density over the projection plane (not evenly distributed over the simulated surfaces). A fixation cross that subtended $3.2^{\circ} \times 3.2^{\circ}$ of visual angle was placed at the center of the monitor. The whole stimulus had a width of $82^{\circ}$ and a height of $52^{\circ}$. A monocular line composed of 50 random dots of the same luminance as the random dots of the planar surface was presented $11.4^{\circ}$ to the left or to the right of the fixation cross. The center of the line was aligned horizontally with the fixation cross, and the vertical separation between the two extremes of the line was $12.7^{\circ}$. The tilt of the line was varied by varying the horizontal separation between the two extremes of the line from a maximum negative separation of $-54.8 \operatorname{arcmin}\left(-4.1^{\circ}\right.$ tilt with respect to the vertical axis) to a maximum positive separation of $54.8 \mathrm{arcmin}\left(4.1^{\circ}\right.$ tilt with respect to the vertical axis). The monocular line was presented together with the binocularly viewed surface for $70 \mathrm{msec}$, to make sure that eye saccades could not be performed.

\section{Procedure}

The observers' task was to judge the tilt of the line. They were instructed to judge whether the top extreme of the line appeared to be on the left or on the right with respect to the bottom extreme of the line. They responded by pressing the left or the right button on the mouse. The observers' responses initiated the following trials. They were asked to keep fixation on the cross in the center of the screen. The experimental room was dark during the experiment, and no feedback was given. 

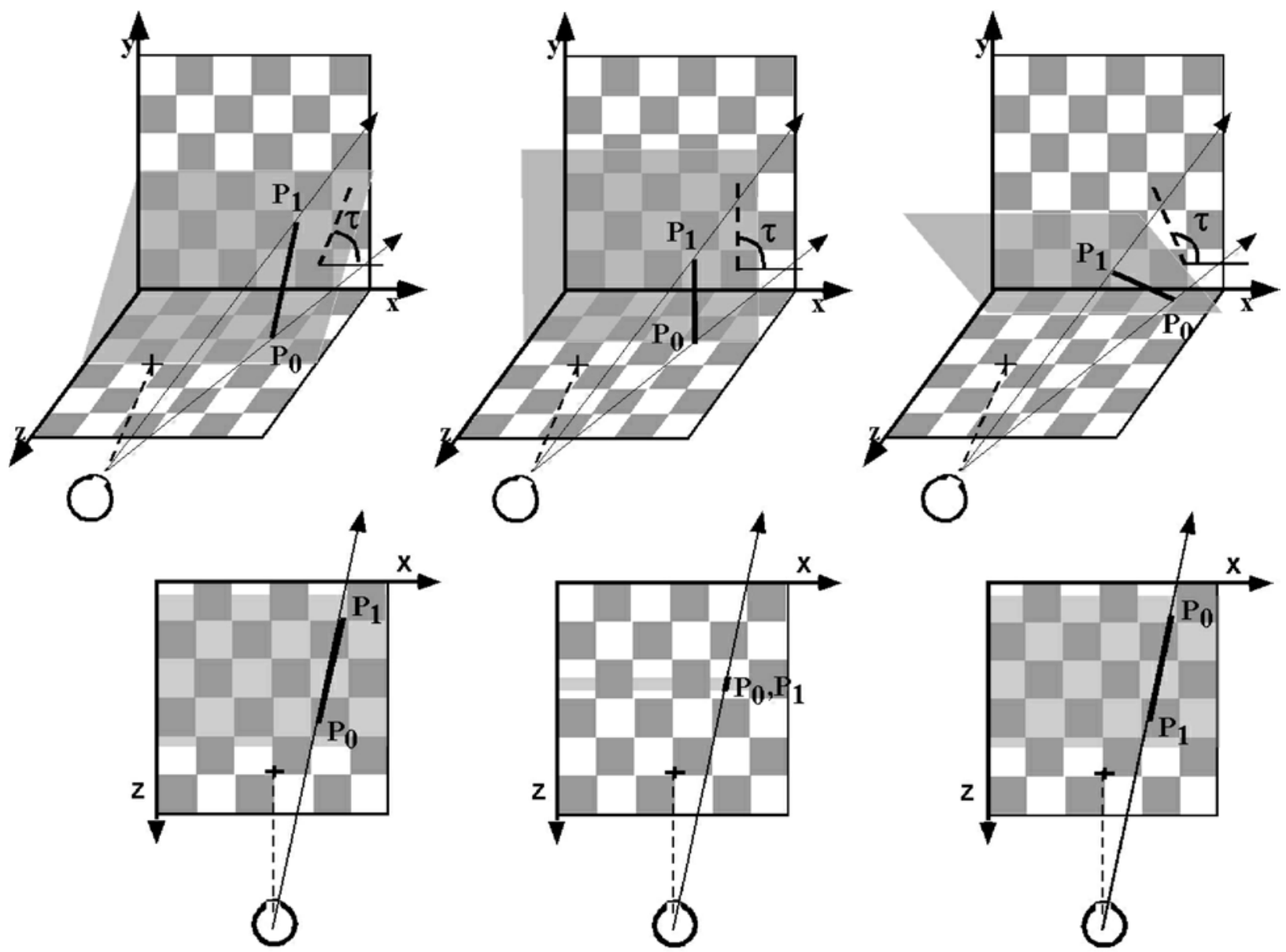

Figure 4. Schematic sketch that illustrates how the perceived tilt of a three-dimensional segment depends on the perceived relative depth between the two extremes of the segment (top panels). In the bottom panels, it can be noted that a different tilt can be perceived even though the perceived visual directions of the two extremes of the segment are the same. The angles in the bottom panels are not intended to be quantitative estimates of visual direction (see the General Discussion section).

\section{EXPERIMENT 1}

The purpose of this experiment was twofold. First we investigated the influence of a slanted surface specified by a stereo stimulus on a monocurlarly viewed randomdot line. As Figures 4 and 5 illustrate, if the line is perceived as part of the surface, its perceived tilt should be biased according to the slant of the surface. Second, we studied the temporal characteristics of the influence of the surface on the perception of the line. The sequence of events is shown in Figure 6. After a fixation cross was presented for $300 \mathrm{msec}$, a random-dot surface was shown for a time interval stimulus onset asynchrony (SOA) that we varied in the range of $0-280 \mathrm{msec}$. Subsequently, a random-dot line appeared either to the left or to the right of the fixation cross for $70 \mathrm{msec}$, together with the surface.

\section{Design}

Five independent variables were examined: the horizontal separation between the top and the bottom extremes of the line $(-54.8$,
$-27.4,0,27.4,54.8$ arcmin), the position of the line (left visual field, $11.4^{\circ}$ to the left of fixation, or right visual field, $11.4^{\circ}$ to the right of fixation), slant of the surface $\left(-33^{\circ}, 0^{\circ}\right.$, or $\left.+33^{\circ}\right)$, SOA of the surface without the line $(0,70,140,210$, or $280 \mathrm{msec})$, and which eye saw the monocular line (left or right). The variables were run within observers, and each of the 300 conditions was repeated 40 times, for a total of 12,000 trials. The 0 -msec SOA was run in the first two sessions. The 70-, 140-, 210-, and 280-msec SOAs were run in a random order within each of the remaining eight sessions. The eye and SOA variables were run in a counterbalanced order between blocks.

\section{Results and Discussion}

The first step in our data analysis was to fit a psychometric function relating the percentage of "top on the right" responses to the horizontal separation between the top and the bottom extremes of the line, in each experimental condition. This nonlinear fit was performed by minimizing the sum of the squared errors between the predicted and the observed data. Two free parameters were used in the fit: the $50 \%$ point, which we will call the bias, and the slope of the psychometric function. If the 


\section{Stimulus}
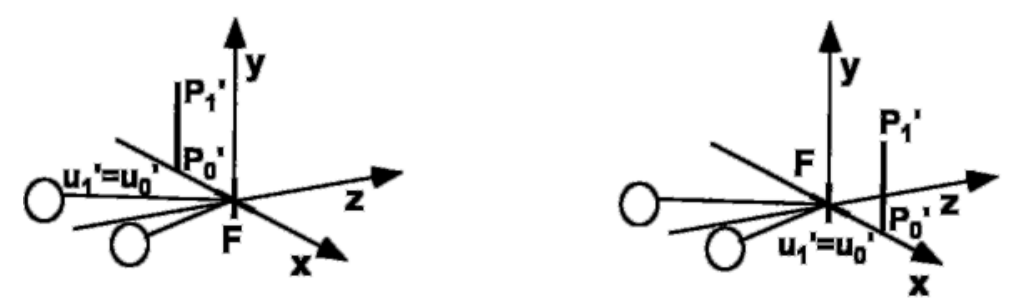

\section{Percept}
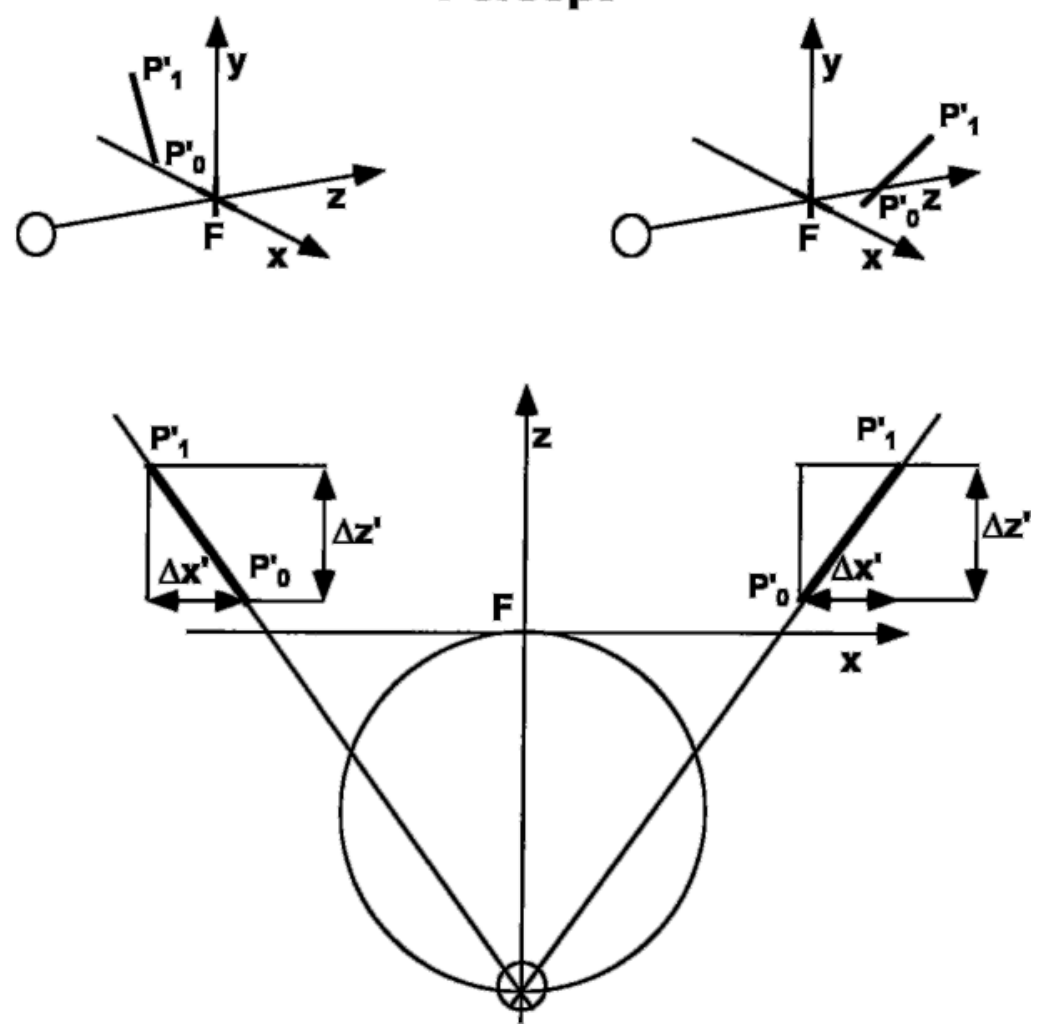

Figure 5. The two top rows sketch, respectively, the simulated and the perceived tilts of a segment presented in the left and right visual fields. The prediction refers to a segment that is perceived as slanted in depth so that the top extreme of the segment is farther away from the observer than the bottom extreme. The bottom panel represents the view of the segment from above.

line is perceived as located on the surface, we should expect that the tilt of the line depends on the slant of the surface and the visual field in which the line is presented. As is sketched in Figure 5, if the slant of the surface is positive, there should be a bias in seeing the top of the line on the right if the line is in the right visual field (corresponding to a shift of the psychometric function toward the left with respect to the 0 -slant condition) and on the left if the line is in the left visual field (corresponding to a shift of the psychometric function toward the right with respect to the 0 -slant condition). These biases should be opposite if the slant is negative. Figure 7 shows the fit of the psychometric functions for an SOA of 0 and $280 \mathrm{msec}$ for subject J.N. ${ }^{3}$ Let us first consider the condition in which the surface was not present before the line appeared ( 0 -msec condition). The shift of the psychometric function with respect to the control condition (in which the surface is frontoparallel) is positive in the left visual field and negative in the right visual field if the surface slant is $+33^{\circ}$. These shifts have opposite signs if the surface slant is $-33^{\circ}$. Therefore, in the $0-m s e c$ condition, the biases measured by the psychometric functions reveal that the tilt of the line was perceived as if the line had the same orientation in depth as the surface. For all $3 \mathrm{ob}-$ 


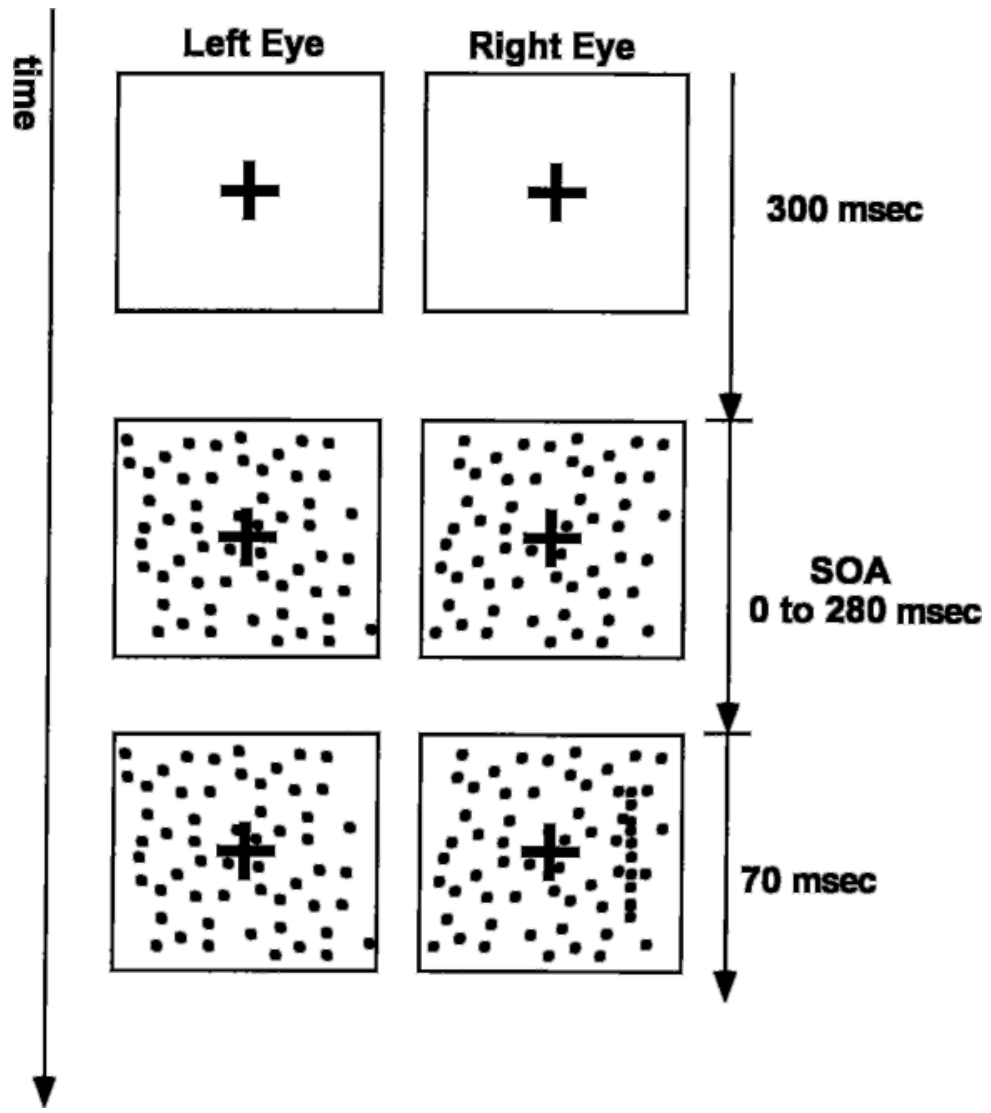

Figure 6. Stimulus sequence in Experiment 1.

servers, this bias almost disappeared when the presentation of the line together with the surface was preceded by the surface alone for $280 \mathrm{msec}$.

In a second stage of the data analysis, we measured the influence of the 3-D surface on the perception of the tilt of the line (1) by calculating the biases (defined as the $50 \%$ point) of the psychometric functions in each experimental condition and (2) by calculating the difference between the biases measured in the right and left visual fields. It can be shown (see the Appendix) that if the line is perceived as located on the surface, this difference is an increasing function of the tangent of the slant of the surface. A 2 (eye) $\times 3$ (slant) $\times 5$ (SOA) within-subjects analysis of variance (ANOVA) was performed on the bias difference between the right and the left visual fields. There was a significant effect of slant $[F(2,4)=11.98$, $p<.05]$ and a significant interaction between slant and SOA $[F(2,4)=13.139, p<.01]$. No other main effects or interactions reached significance. The plot of Figure 8 shows the difference between the biases of the right and the left visual fields as a function of the slant of the surface for each SOA and for each observer. It can be noticed that the slope of this function is a decreasing function of the SOA and that this reduction is already substantial for an SOA of $70 \mathrm{msec}$. Figure 9 clearly shows how the slope of the function that relates the bias difference to the slant of the surface decreases with the SOA.

In conclusion, the results of the first experiment suggest that a 3-D stereo surface can influence the perceived tilt of a monocular line. The observed biases are compatible with the hypothesis that the line is perceived as being located on the 3-D surface. However, this effect almost disappears when the surface is presented prior to the appearance of the monocular line.

\section{EXPERIMENT 2}

The second experiment was motivated by the finding that, for all observers, even $70 \mathrm{msec}$ of preexposure to the surface are sufficient to substantially reduce the influence of the stereo surface on the perceived tilt of the monocular line: After $70 \mathrm{msec}$, the effect of SOA seems to be very small. Moreover, the data show substantial individual differences. For these reasons, the SOA variable was sampled in a smaller range $(0-70 \mathrm{msec})$, and more observers (8) participated in the experiment.

\section{Design}

The design was identical to the first experiment, except that only two slants were simulated $\left(-33^{\circ}\right.$ or $\left.+33^{\circ}\right)$, the SOA range was 0 , 


\section{Left Visual Field Right Visual Field}

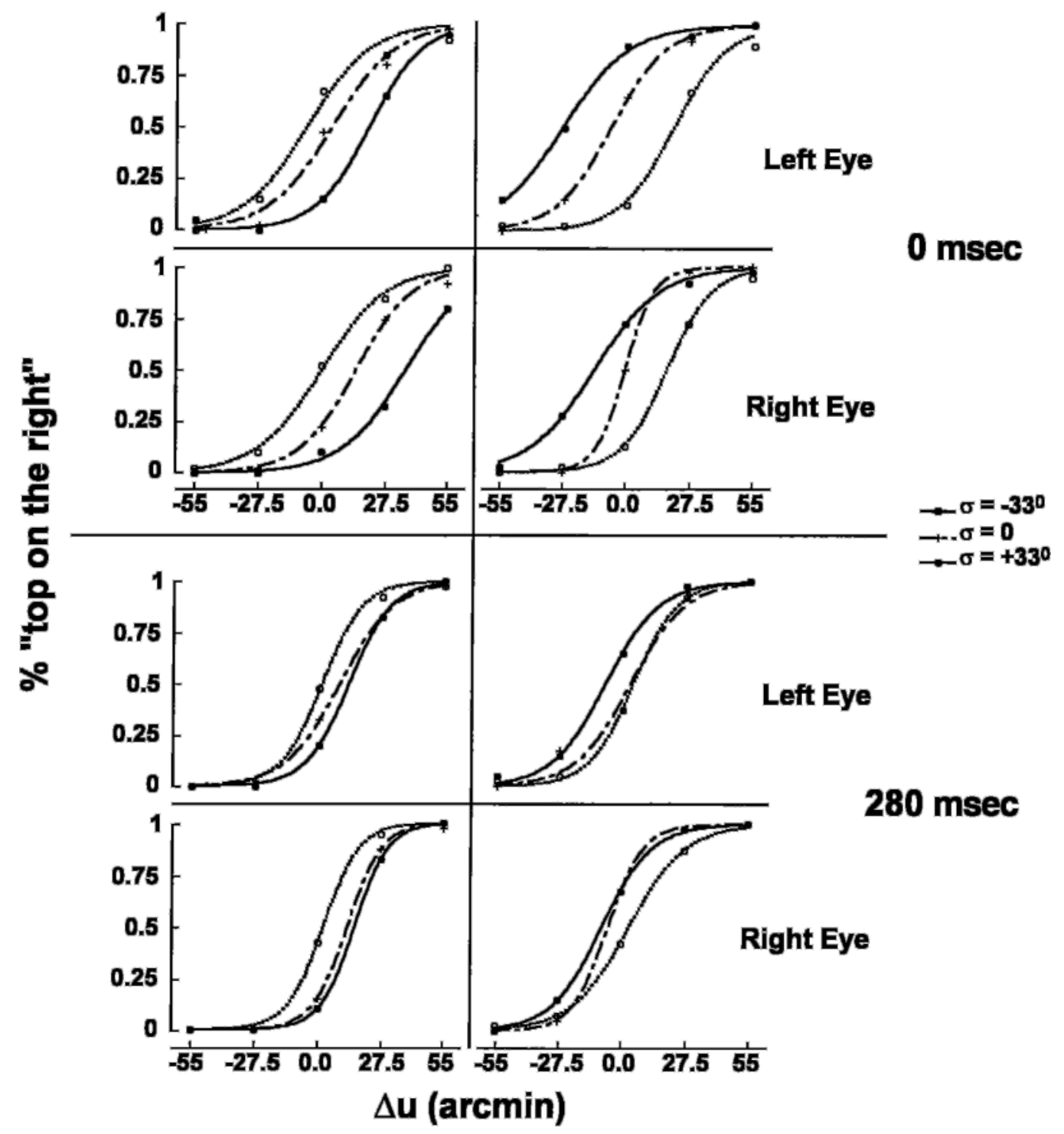

Figure 7. Psychometric functions for the three simulated slants in two experimental conditions of Experiment 1 for the left and the right eyes of observer J.N. and for the left and right visual fields. The $x$-axis represents the horizontal separation between the two extremes of the line on the image plane. The $y$-axis represents the percentage of times in which the top extreme of the monocular line was perceived on the right.

23,46 , or $70 \mathrm{msec}$, and the eye viewing the monocular line was a between-subjects variable.

\section{Results and Discussion}

As in the previous experiment, we measured the influence of the 3-D surface on the perception of the twodimensional (2-D) orientation of the line by calculating the difference between the biases measured in the right and the left visual fields in each experimental condition. A 2 (eye) $\times 2$ (slant) $\times 4$ (SOA) within-subjects ANOVA was performed on the bias difference between the right and the left visual fields. There was a significant effect of slant $[F(1,6)=14.17, p<.01]$ and a significant interaction between slant and SOA $[F(2,4)=4.663, p<.05]$. No other main effects or interactions reached significance. Moreover, we calculated the slope of the function that relates the bias difference between the right and the left visual fields to the slant of the surface for each SOA and for each observer. A 2 (eye) $\times 4$ (SOA) ANOVA was performed on the slope. There was a significant effect of SOA $[F(3,18)=4.66, p<.05]$. No other main effects or interactions reached significance. Figure 10 shows how the slope of the function that relates the bias difference to the slant of the surface decreases with the SOA. 


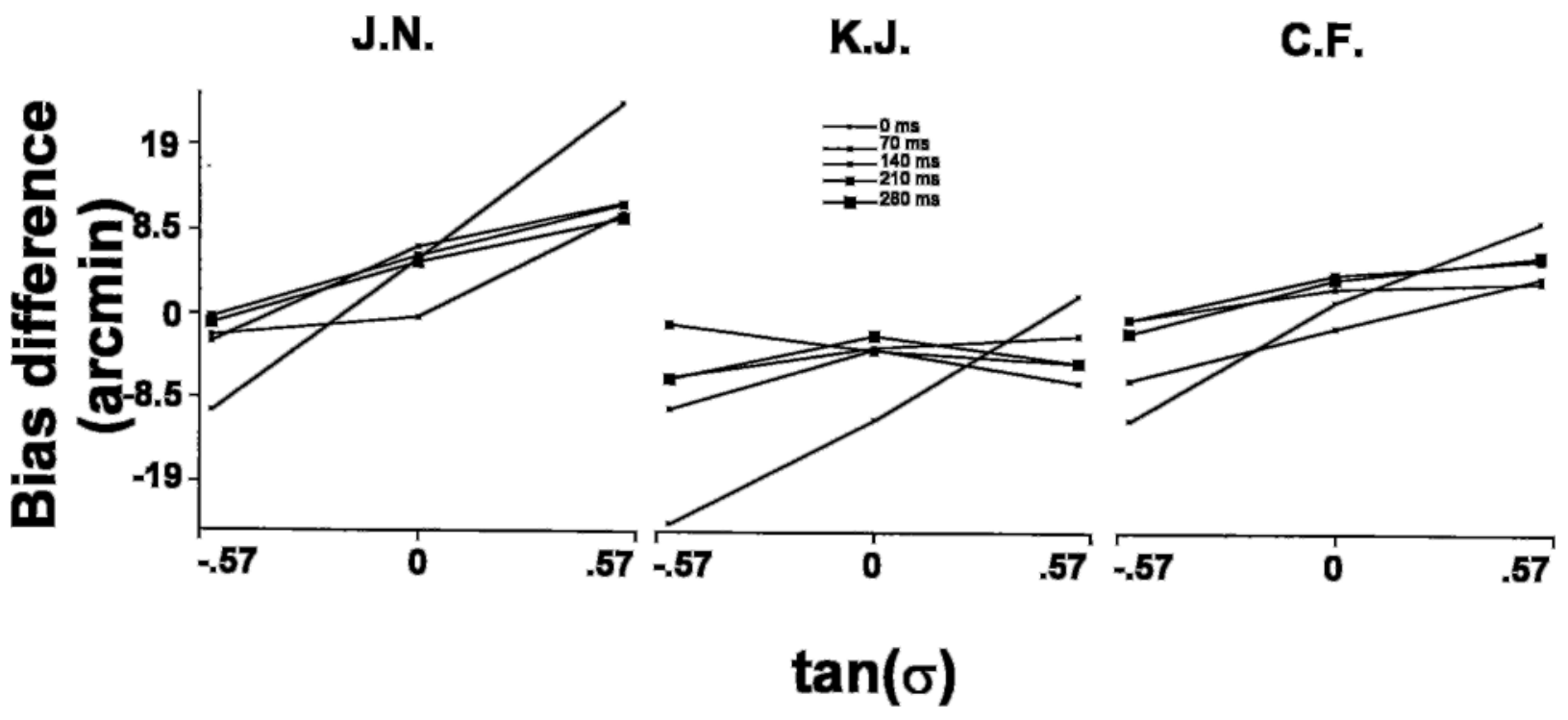

Figure 8. Difference between the thresholds of the psychometric functions for right and left visual fields as a function of the tangent of the slant of the surface for each observer. Different curves refer to different stimulus onset asynchronies.

The results of the second experiment replicate the major findings of the first experiment, since they show that (1) the perceived tilt of the monocular segment is influenced by the slant of the stereo surface and (2) this influence depends on the prior presentation of the surface. Moreover, these results indicate that, on average, $46 \mathrm{msec}$ of preexposure to the stereo surface is sufficient to significantly reduce the effect.

\section{EXPERIMENT 3}

The effect of preexposure to the surface found in Experiments 1 and 2 shows that temporal synchronicity between presentation of the surface and presentation of the monocular line is an important factor for maximizing the influence of the 3-D surface on the perceived tilt of the monocular line. These results may indicate that when line and surface appeared at different instants of time, they were considered as distinct objects. On the other hand, when they were flashed together briefly without prior exposure of the stereo surface, a "fusion" of monocular line and stereo surface may have been experienced.

The purpose of the present experiment was to investigate whether the influence of the surface on the monocular line is still inhibited when a blank stimulus follows the initial presentation of the stereo surface and whether a sufficiently long blank stimulus can reestablish the "fusion" between the 3-D surface and the monocular line. To answer these empirical questions, we created the stimulus sequences depicted in Figure 11. In one condition, the stereo surface alone was presented for $300 \mathrm{msec}$ (prior presentation condition, PP). This presentation was followed by a blank interval of either 100 or $600 \mathrm{msec}$ (interstimulus interval, ISI). Then, the line and the surface were flashed together for $70 \mathrm{msec}$. The other condition was identical, except that the prior presentation of the stereo surface was replaced by the fixation mark (no prior presentation condition, NPP). If the mechanism that inhibits the influence of the 3-D surface on the perceived 2-D orientation of the line is still active even when the stereo surface is no longer present, we should expect that a short ISI of $100 \mathrm{msec}$ will not be sufficient to reestablish the "fusion" of monocular line and surface. However, a long enough ISI $(600 \mathrm{msec})$ may be sufficient to inactivate such an inhibitory mechanism.

\section{Design}

Six independent variables were examined: the horizontal separation between the top and the bottom extremes of the line $(-54.8$, $-27.4,0,27.4$, or 54.8 arcmin), position of the line $\left(11.4^{\circ}\right.$ to the left of fixation or $11.4^{\circ}$ to the right of fixation), slant of the surface $\left(-33^{\circ}\right.$ or $\left.+33^{\circ}\right)$, prior presentation of the surface (PP or NPP), ISI between the prior presentation of the surface (in the NPP condition, ISI was an extension of the time during which the fixation cross was present on the screen) and the presentation of the surface and the line (100 or $600 \mathrm{msec}$ ), and the eye that saw the monocular line (left or right). The variables were run within observers, and each experimental condition was repeated 40 times, for a total of 6,400 trials. The observers were run in four sessions of four blocks each. The Prior Presentation and ISI variables were run between sessions. The eye variable was run between blocks.

\section{Results and Discussion}

As in the previous experiment, we measured the influence of the 3-D surface on the perception of the tilt of the line by calculating the difference between the biases measured in the right and the left visual fields in each experimental condition. A 2 (eye) $\times 2$ (slant) $\times 2$ (ISI) $\times 2$ (prior presentation) within-subjects ANOVA was performed on the bias difference between the right and the left visual fields. There was a significant effect of slant $[F(1,4)=28.87, p<.01]$, a significant interaction be- 


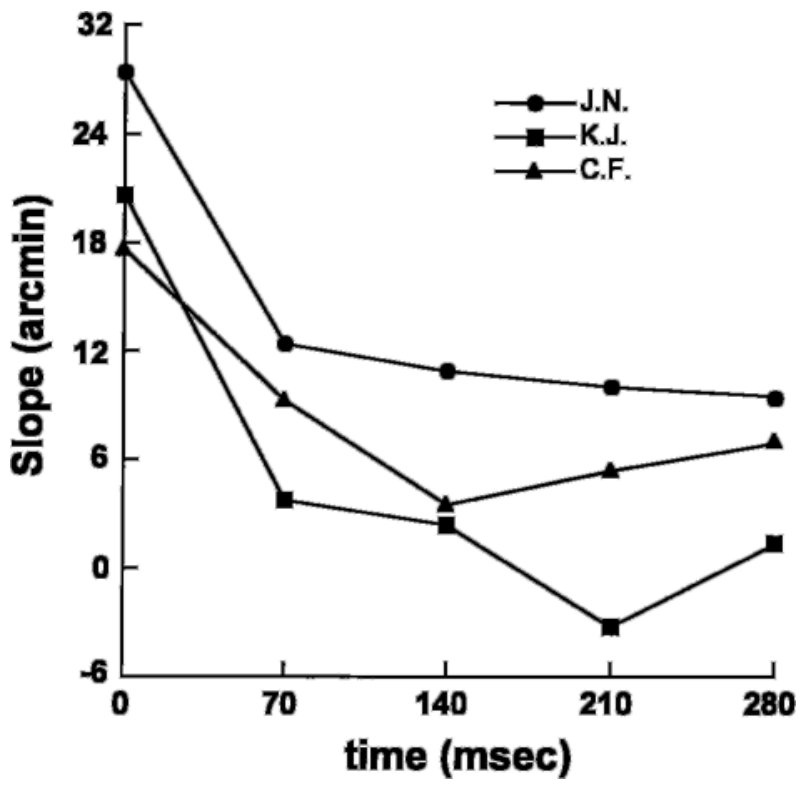

Figure 9. Slope of the curves plotted in Figure 8 as a function of stimulus onset asynchrony for each observer.

tween slant and SOA $[F(1,4)=13.43, p<.05]$, and a significant interaction between slant, prior presentation, and ISI $[F(1,4)=9.975, p<.05]$. No other main effects or interactions reached significance.

The effect of slant replicates the findings of the previous experiment, since it indicates that the 3 -D surface influences the perceived tilt of the monocular line. To interpret the significant interactions, let us consider Figure 12, in which the slope of the function relating bias difference (see the analysis of Experiment 1) and the tangent of the slant of the surface is plotted against ISI for the naive observers and for 1 knowledgeable observer, F.D. As was described in the previous experiment, if the line is perceived as being located on the surface, the bias difference is a linear increasing function of the tangent of the slant of the surface. When the ISI is $100 \mathrm{msec}$, there is a difference between the condition in which there is a prior presentation of the stereo surface and the condition in which there is no prior presentation of the surface. When there is a prior surface presentation (PP condition), there is a reduction in the effect of the 3-D surface on the perceived orientation of the monocular line. This means that the effect of prior presentation of a stereo surface that we found in the previous experiment is still present after a 100 -msec period during which the stereo surface is absent and a blank stimulus is viewed instead. However, when the ISI is $600 \mathrm{msec}$, prior presentation of the stereo surface has little influence on the effect of the 3-D surface on the perceived orientation of the monocular line. Figure 13 shows an overview of the results for each naive observer. It can be noticed that 1 observer (G.S.) does not show the interaction between ISI and SOA. A possible explanation of this result is that, for this observer, the effect of prior presentation of the stereo surface lasts for less than $100 \mathrm{msec}$.

In summary, these results indicate that the influence of a 3-D surface on the perceived orientation of a monocular line is inhibited by a previous presentation of the 3-D surface alone even when a 100-msec blank follows the prior presentation of the surface, implying that this inhibitory mechanism lasts, on average, for at least $100 \mathrm{msec}$. On the other hand, a blank interval of $600 \mathrm{msec}$ is sufficient to substantially reduce the effect of prior presentation of the stereo surface.

\section{GENERAL DISCUSSION}

The main purpose of the three experiments described in the present paper was to determine the influence of a stereo-specified planar surface on the perception of a monocular line. We reasoned that if a monocular line, which does not provide any cue to depth, is embedded in a random-dot disparity field that specifies a 3-D planar surface, the line may be perceived as belonging to the surface in which it is embedded. If this is the case, the perceived tilt of the line should be related to the slant of the binocular surface, as is predicted by perspective geometry.

The results of the experiments indicate that the perceived orientation of the monocular line is influenced by the slant of the 3-D surface. Moreover, the results reveal that this influence depends on the duration of a previous exposure to the binocular surface. If the surface and the line are presented together without prior exposure to the surface, the influence of the surface on the line is much larger then if the presentation of the surface precedes the presentation of the line. We will discuss these two main findings below, in an attempt to explain the spatial and temporal properties of the phenomenon.

The fact that the perceived tilt of the monocular line is influenced by the slant of the surface can be explained by means of perspective geometry. If the line is perceived as being located on the surface, the perceived tilt of the line should depend on the slant of the surface (see Figures 4 and 5), as the results of Experiments 1 and 2 indicate. This result is similar to a result that we have found in a study with monocular stimuli (Domini \& Braunstein, 1998). In that study, the observers saw a random-dot planar surface rotating in depth, and at the end of the SFM sequence, a pair of dots was flashed for a brief interval. The task of the observer was to judge the tilt of the imaginary line connecting the dots. As we found in the present study, the perceived tilt of the imaginary line depended on the perceived slant of the SFM surface, and the effect could be predicted by the laws of perspective geometry.

The fact that the same effect has been found in experiments in which 3-D surfaces were specified by different sources of information (stereo and motion) may suggest that the phenomenon is general enough and should not be attributed to the specific source of information under 


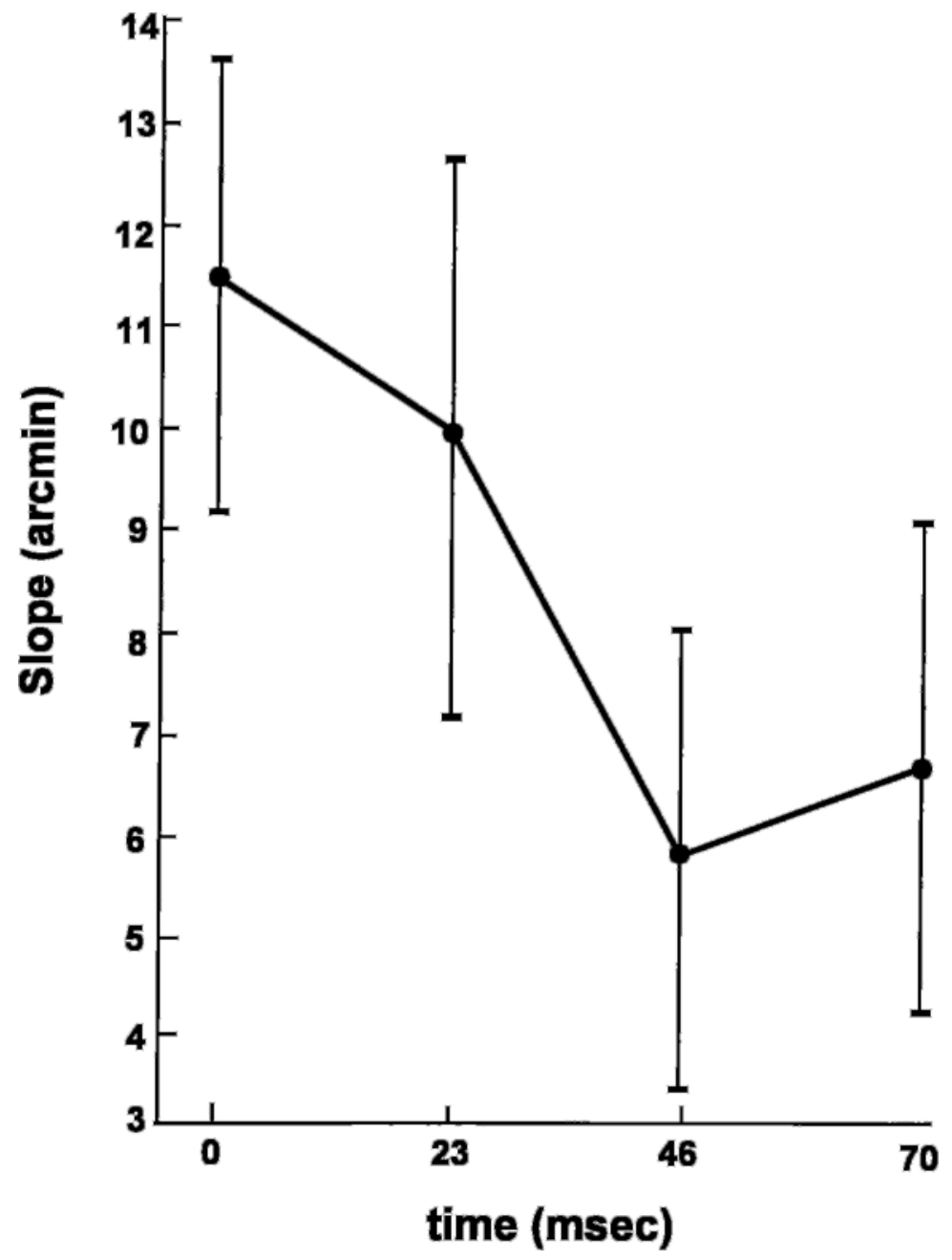

Figure 10. Average slope of the function that relates the bias difference between the right and the left visual fields to the slant of the stereo surface for each stimulus onset asynchrony in Experiment 2.

study. However, whereas in monocular vision the visual direction of a target is completely specified by the position of the target with respect to the viewing eye, in stereovision visual direction must be computed with respect to the cyclopean eye, and information from both eyes must be combined. We should therefore analyze how different predictions about perceived visual direction may affect the interpretation of the present results.

Hering's (1879/1942) law predicts that the visual direction of a monocular target depends only on the position of the target with respect to the viewing eye. Figure 14 sketches Hering's predictions: If, say, the right eye sees the monocular target (Figure 14, top left), the angle $\alpha_{\mathrm{R}}$ between the visual axis and the visual line determines the perceived visual direction (Figure 14, top right). This law would therefore predict that the stereo surface does not influence the perceived visual direction of the monoc- ular line. Hering's law, therefore, cannot explain the present findings.

However, the view that the perceived visual direction of a monocular element is independent on the binocular context in which it is embedded has been questioned recently. In fact, Erkelens and van de Grind (1994), Erkelens et al. (1996), Erkelens and van Ee (1997a), and van Ee, Banks, and Backus (1999b) have reported that perceived directions of monocular objects are captured by adjacent binocular objects. In particular, their experiments have revealed that the perceived relative directions of monocular targets are consistent with the directions of the eye that sees both targets. Figure 15 shows Erkelens and van Ee's prediction for the perceived relative directions of the two extremes of the monocular line segment. Let us first consider the case in which the right eye sees the monocular segment (top left panel). The extremes of the 


\section{Prior Presentation Non Prior Presentation}

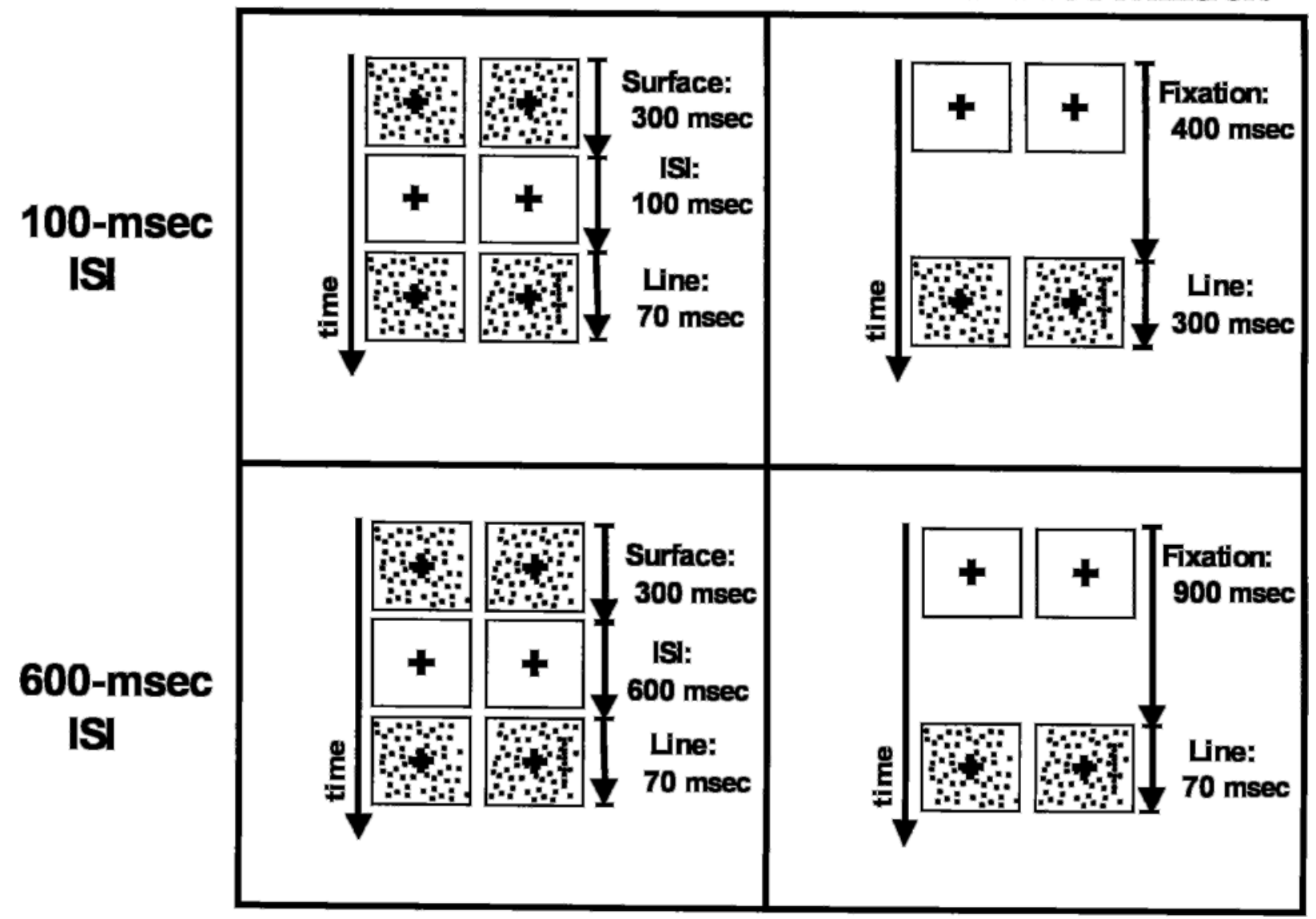

Figure 11. Stimulus sequence in Experiment 3.

segment, represented by the points $\mathrm{P}_{0}$ (bottom) and $\mathrm{P}_{1}$ (top), will be next to binocular elements $\mathrm{B}_{0}$ and $\mathrm{B}_{1}$ that belong to the 3-D surface. If the 3-D surface is a floor, for example, then $\mathrm{P}_{0}$ will be adjacent to a binocular element $B_{0}$ that is closer to the observer than $B_{1}$, the binocular element adjacent to $P_{1}$. If the perceived visual directions of the monocular elements are captured by the perceived visual directions of the binocular elements, the perceived visual direction of the extreme $P_{1}$ will be on the left of the perceived visual direction of the extreme $\mathrm{P}_{0}$ (top right panel). On the other hand, the perceived relative directions of the segment extremes will have an opposite spatial ordering if the eye that sees the monocular line is the left eye. These predictions are not incompatible with our results, but they cannot explain them. If the perceived tilt of the line was merely based on the perceived visual directions, we would expect opposite results for the two eyes (specifically, a leftward tilt for the left eye and a rightward tilt for the right eye). However, the results do not show any significant effect of the eye of origin. Our prediction, on the other hand, is that the perceived tilt does not depend only on the perceived visual direction of the two extremes, but also on the slant of the stereo surface on which the line is superimposed. If the points $P_{0}$ and $P_{1}$ in Figure 15 are perceived as being located at $\mathrm{B}_{0}$ and $\mathrm{B}_{1}$, respectively, $\mathrm{P}_{1}$ should be perceived on the right of $\mathrm{P}_{0}$ independently of the viewing eye. Our results are compatible with this prediction.

In another study that may be considered closely related to the present investigation, Rogers and Bradshaw (1999) found that when two monocular nonius lines aligned on the image plane are embedded in a disparity field specifying a surface slanted about the $x$-axis, they are perceived as having different 2 -D orientations. The authors attribute this effect to the fact that two monocular images created by an inclined surface are related by a horizontal shear transformation. In other words, a group of local elements in the right eye image are, to a first approximation, rotated clockwise with respect to the corresponding elements on the left eye image if the projected surface is a "floor." They therefore hypothesized that if a monocular element projects as a vertical line, its orientation should be perceived in reference to the binocular elements in which it is embedded. A vertical monocular line in the right eye should therefore be perceived as being rotated counterclockwise, since the frame of reference 


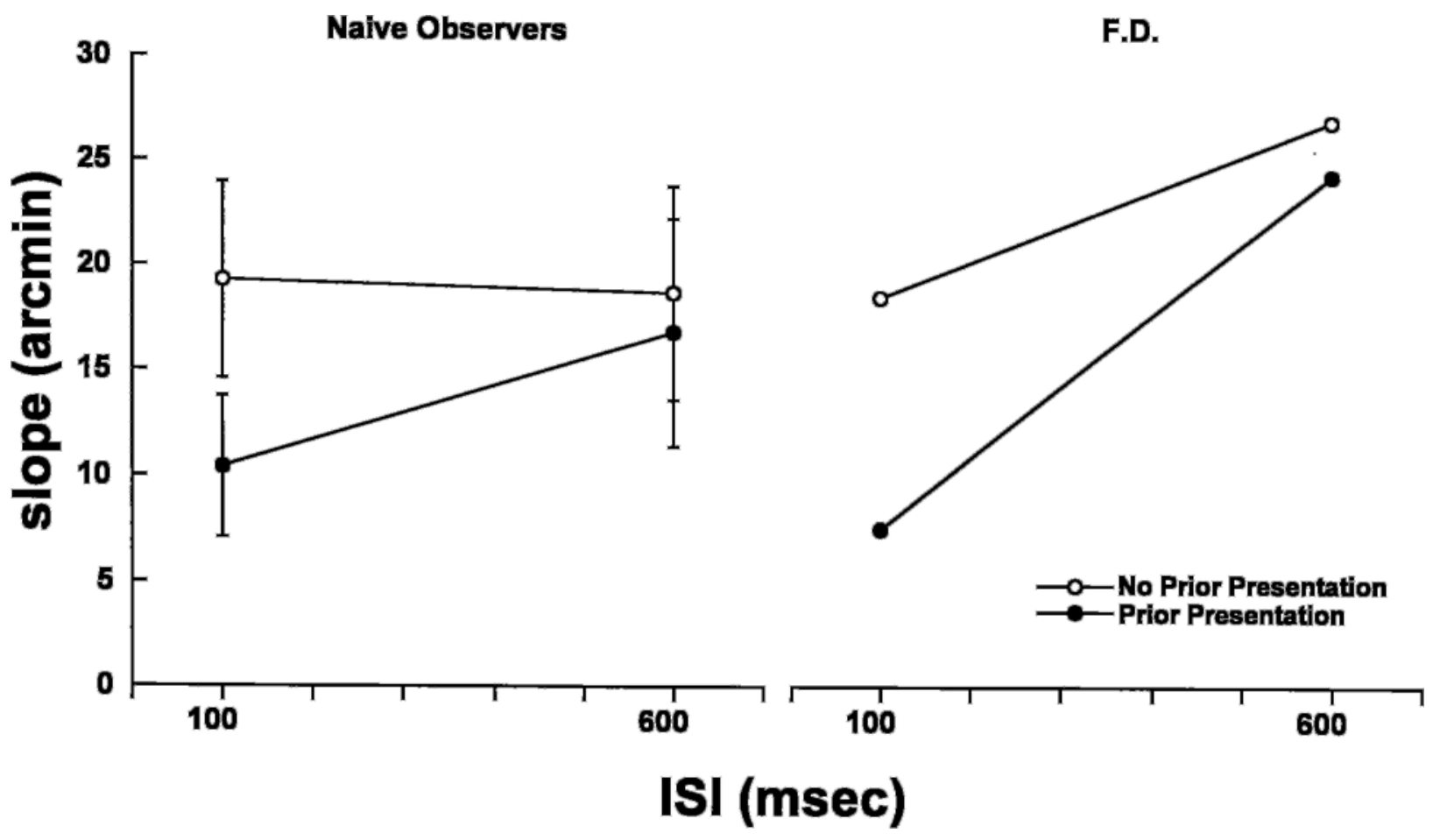

Figure 12. Slope of the function relating the threshold difference for the two visual fields and the tangent of the slant plotted against interstimulus interval (ISI) in Experiment 3. The two curves represent conditions in which there was no prior presentation of the surface and in which there was a prior presentation. Left and right panels show the average results for 4 naive observers and 1 knowledgeable observer.

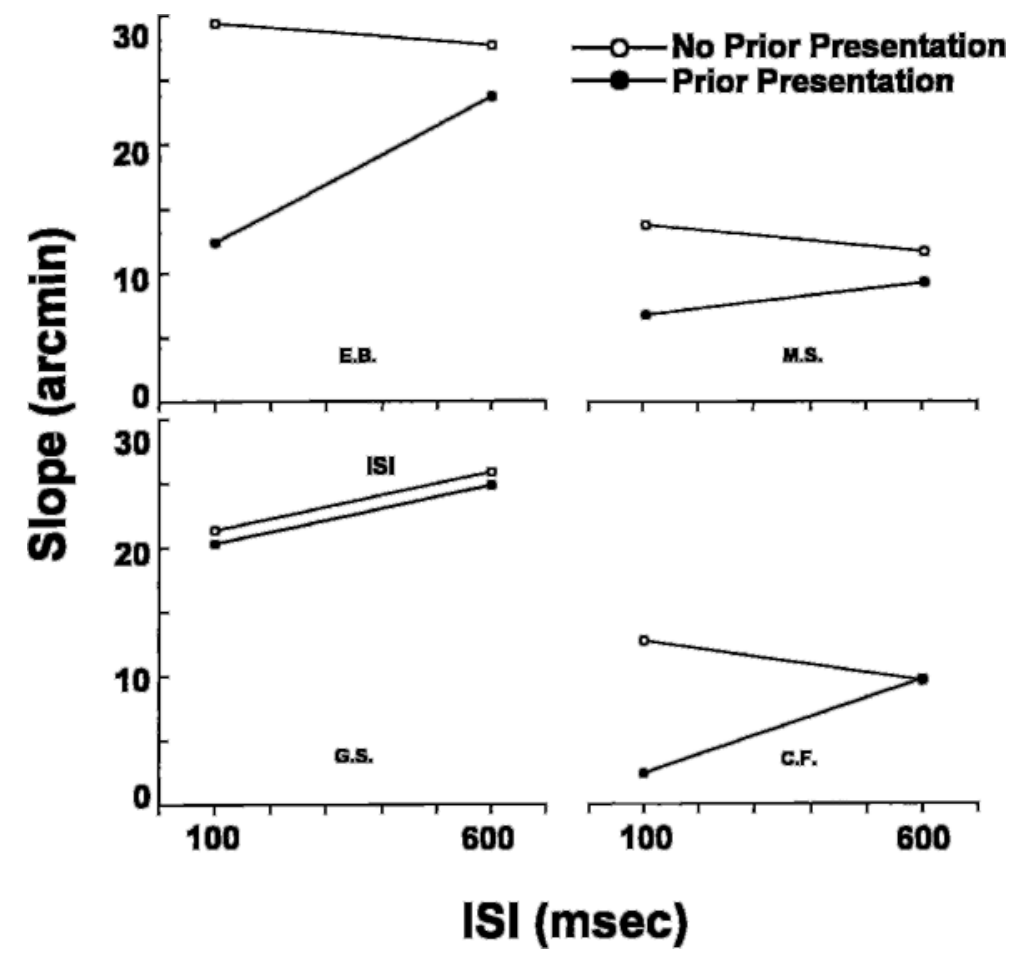

Figure 13. Results of Experiment 3 for each individual observer. 

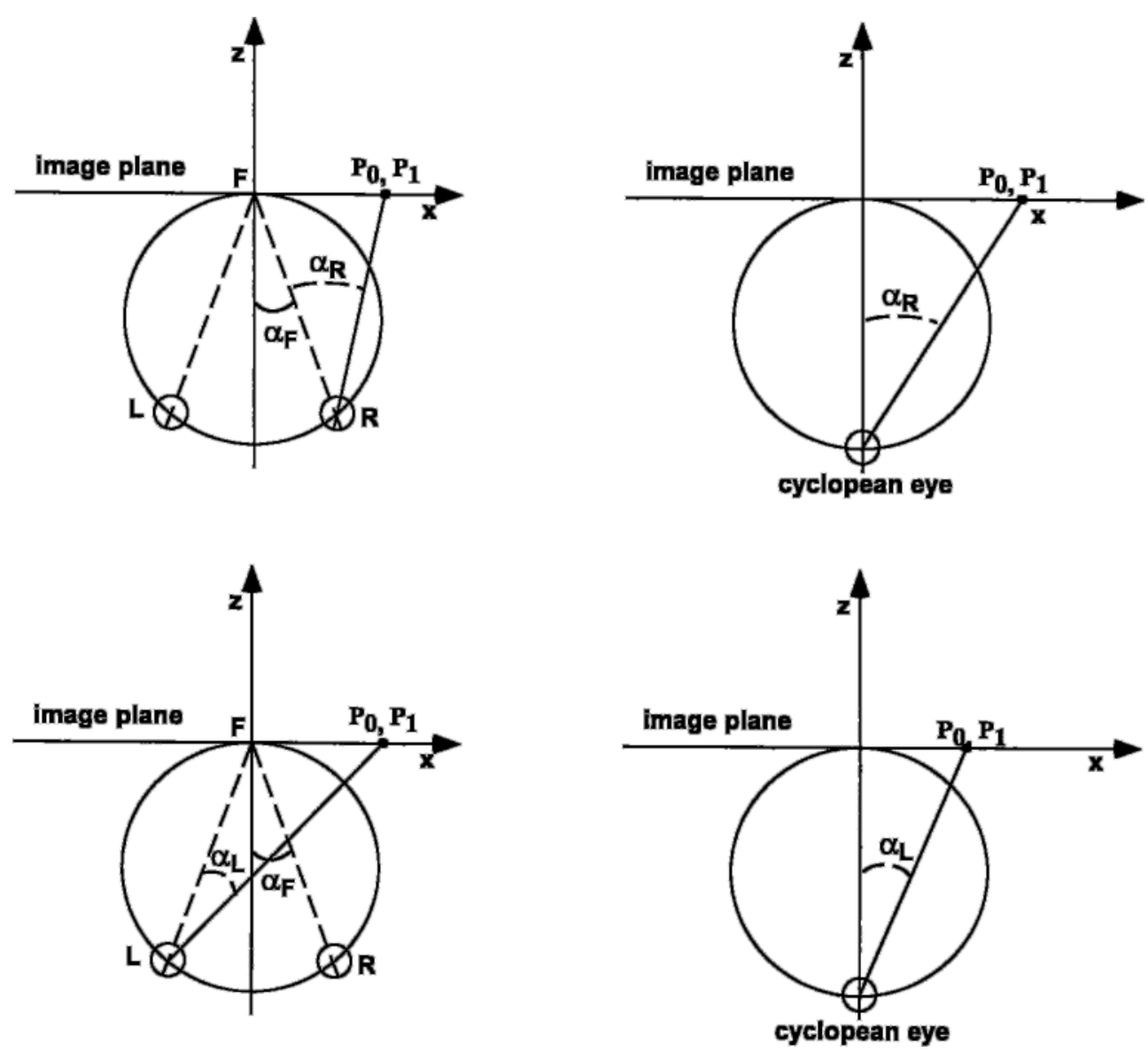

Figure 14. Hering's (1879/1942) predictions about perceived visual direction of the two extremes $\left(P_{0}\right.$ and $\left.P_{1}\right)$ of the monocular line segment when it is presented to the right visual field and is viewed by the right eye (top panels) and the left eye (bottom panels).

constituted by the binocular right-eye elements is rotated clockwise. For the same reason, an opposite effect should be expected for the opposite eye. This fact can be interpreted as evidence that the relative position of monocular stimulus elements with respect to the binocular stimulus elements for the same eye is maintained in perception.

These results should be considered different from the results described in the present paper, since in Rogers and Bradshaw's (1999) study, the perceived orientation of the monocular line is contingent on the eye of origin. In our study, on the other hand, we hypothesize that the perceived orientation of the line depends on the visual field in which it is presented. Since we compare the perceived 2-D orientation of the line in the two visual fields, any effect of the eye of origin is automatically filtered out. In fact, if there is any bias to see a vertical line presented on the right eye as being rotated toward the left, this bias should be present in both visual fields. Instead, what the results of Experiments 1 and 2 show is that a monocular line on the right visual field has a rightward orientation with respect to a monocular line presented in the left vi- sual field if the lines are embedded in a disparity field specifying a "floor" surface. The difference between the perceived orientation of the line in the two visual fields can therefore not be attributed to the mechanism revealed by Rogers and Bradshaw's results. We suggest that the results of Experiments 1 and 2, together with the experiment of Domini and Braunstein (1998), indicate instead that the perceptual system may locate 2-D elements that do not themselves provide any 3-D cue to depth on the 3-D surface specified by the region in which they are embedded.

Although our experiments were not designed to test Rogers and Bradshaw's (1999) hypothesis, they can be analyzed for consistency with their hypothesis. We examined the mean thresholds of the psychometric functions (in minutes of arc) for six conditions in Experiment 1. An increase in the mean indicates an increasing bias in seeing the line tilted counterclockwise. Following Rogers and Bradshaw's reasoning, when the surface orientation changes from ceiling to floor, the elements in the right eye rotate clockwise. Therefore, monocular elements should seem to rotate in the opposite direction, since their per- 

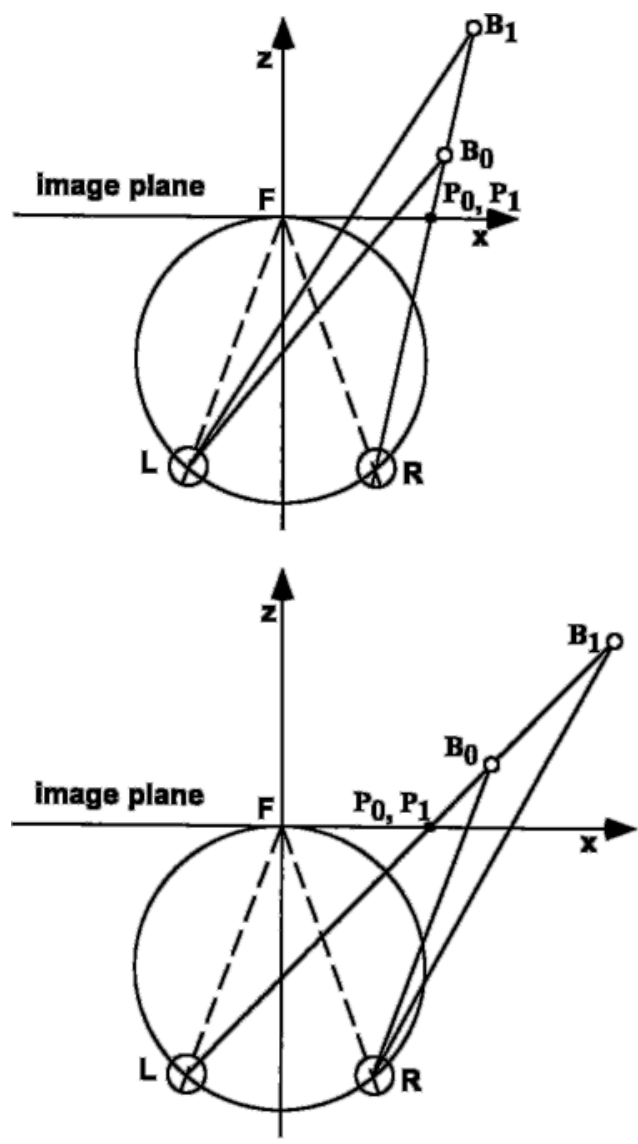
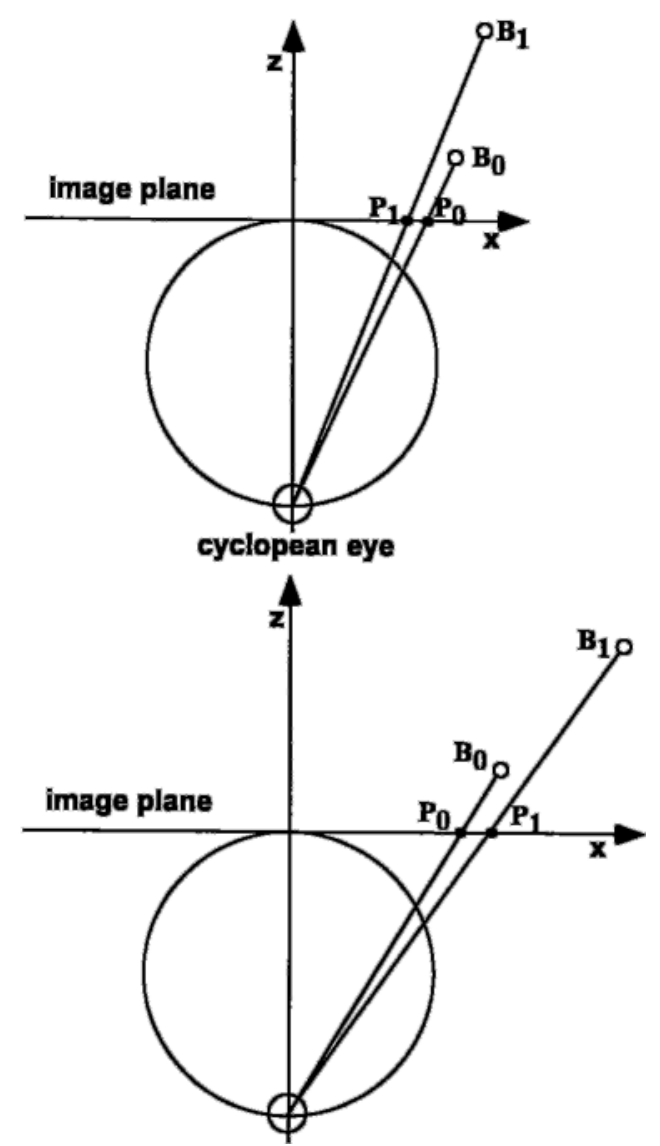

Figure 15. Erkelens's predictions about perceived visual direction of the two extremes $\left(P_{0}\right.$ and $\left.P_{1}\right)$ of the monocular line segment when it is presented to the right visual field and is viewed by the right eye (top panel) and the left eye (bottom panel).

ceived 2-D orientation is relative to the background (opposite prediction for the left eye). Our results are fully consistent with their hypothesis. For the right eye we obtained means of $4.58,4.88$, and 5.46 for the $-33^{\circ}, 0^{\circ}$, and $33^{\circ}$ surface slants ("ceiling," frontal, and "floor" conditions), whereas for the left eye we obtained means of 2.53, 2.37, and 2.07 for those conditions.

The spatial configuration of our stimuli is not the only important property that determines the influence of the slanted surface on the perceived orientation of the monocular line. Another factor turned out to be fundamental: timing. When the surface and the line were flashed together and in isolation, a strong influence of the surface on the perceived orientation of the line was observed. However, when the surface alone preceded this stimulus configuration, the effect was substantially reduced. Therefore, a prior exposure to the surface reduced the influence of the surface on the perceived line orientation. These temporal effects resemble those found by Wolfe (1986) in a study of the perception of binocular rivalry. When two potentially rivalrous images like horizontal and vertical lines are flashed for $20 \mathrm{msec}$, they are perceived as a grating and do not produce rivalry, as they do when they are seen for a longer time. As in the case of the surface and the line in the present experiments, the vertical and horizontal patterns are therefore perceived as belonging to the same object-namely, a 2-D grating. When the presentation of the two patterns is preceded by the presentation of any stereo stimulus, abnormal fusion is not experienced anymore, but rivalry is experienced instead. In a similar fashion, if the presentation of the surface with the line is preceded by the presentation of the surface alone, the "fusion" between the surface and the line is substantially reduced. For this "fusion" to be reestablished, a sufficiently long blank interval must be introduced between the prior presentation of the stereo surface and the presentation of the surface with the line, as was indicated by the results of the second experiment.

The present data are not sufficient to determine whether or not the temporal effects that we have found can be related to the same rivalry mechanism as that hypothesized by Wolfe (1986). In fact, the monocular line in our experiments always dominated, and therefore, no rivalry was experienced. Nevertheless, the similarity between the 
timing effect that we found and the timing of rivarlry phenomena might be pursued in further research. In general, we can speculate about the existence of a mechanism that uses the temporal onset of ambiguous monocular elements, relative to the unambiguous context in which they are embedded, to determine whether or not to integrate the ambiguous elements into that context. In our experiments, when the surface and the line are flashed together, there is no evidence contrary to the assumption that the line belongs to the surface. Both have the same texture configuration, and the disparity information of the line is ambiguous. Since they appear together and at the same time, the perceptual system might interpret them as being part of the same object. However, when the surface is presented prior to the appearance of the line, the appearance of the line can be considered by the observer as a new event and the line as a new object, not necessarily related to the surface. In this case, the system attributes to the ambiguous 3-D information provided by the line another default 3-D interpretation-that is, that the line belongs to the frontoparallel plane. Evidence that visual synchrony affects perceptual binding and segmentation has been recently provided by Usher and Donnelly (1998). In their study, they found that a lattice of dots arranged in square matrix would be perceived as being organized in columns or rows if alternate columns or rows were presented in successive 16-msec time intervals. On the other hand, the same pattern would be perceived as ambiguous if all the dots were presented simultaneously. It is very important to note that a $16-\mathrm{msec}$ interval of asynchrony could not be consciously detected by the observers that participated in the experiment.

In conclusion, the present results suggest that the perceived orientation of monocular elements embedded in a disparity field is determined by both spatial and temporal properties of the stimulus. Two main findings summarize our results. First, the perceived tilt of a monocular line superimposed on a slanted stereo planar surface can be predicted by perspective geometry as if the line is perceived as being located on the surface. Second, the presentation of the surface alone prior to the presentation of the surface with the line substantially reduces the influence of the stereo surface on the perceived 2-D orientation of the line. This effect may suggest the existence of a heuristic process that uses simultaneity of onset to determine whether or not to combine noncorresponding elements in the two retinal images into a unified percept.

\section{REFERENCES}

Anderson, B. L., \& NAKAYAma, K. (1994). Toward a general theory of stereopsis: Binocular matching, occluding contours, and fusion. Psychological Review, 101, 414-445.

Domini, F., \& Braunstein, M. L. (1998). Structure-from-motion dis- plays can prime perspective distortions [Abstract]. Investigative Ophthalmology \& Visual Science, 39, S1084.

Erkelens, C. J., Muiss, A. J. M., \& VAn Ee, R (1996). Binocular alignment in different depth planes. Vision Research, 36, 2141-2147.

ERKelens, C. J., \& VAN DE Grind, W. A. (1994). Binocular visual direction. Vision Research, 34, 2963-2969.

ERKELENS, C. J., \& VAN EE, R. (1997a). Capture of the visual direction of monocular objects by adjacent binocular objects. Vision Research, 37, 1735-1745.

ERKelens, C. J., \& VAN Ee, R. (1997b). Capture of visual direction: An unexpected phenomenon in binocular vision. Vision Research, 37, 1193-1196.

Hering, E. (1942). Spatial sense and movements of the eye (C. A. Radde, Trans.). Baltimore: American Academy of Optometry. (Original work published 1879)

Howard, I., \& Rogers, B. J. (1995). Binocular vision and stereopsis. New York: Oxford University Press.

Julesz, B. (1960). Binocular depth perception of computer generated patterns. Bell System Technical Journal, 39, 1125-1162.

Kumar, T., \& Glaser, D. A. (1991). Influence of remote objects on local depth perception. Vision Research, 31, 1687-1699.

Kumar, T., \& Glaser, D. A. (1993). Temporal aspects of depth contrast. Vision Research, 33, 947-957.

NaKaYAma, K., \& Shimojo, S. (1990). Da Vinci stereopsis: Depth and subjective occluding contours from unpaired image points. Vision Research, 30, 1811-1825.

OnO, H., \& MAPP, A. P. (1995). A restatement and modification of Wells-Hering's laws of visual direction. Perception, 24, 237-252.

Rogers, B. J., \& Bradshaw, M. F. (1999). Disparity minimisation, cyclovergence, and the validity of nonius lines as a technique for measuring torsional alignment. Perception, 28, 127-141.

Shimojo, S., \& NaKayama, K. (1990). Real world occlusion constraints and binocular rivalry. Vision Research, 30, 69-80.

SHImOJo, S., \& NAKAYAMA, K. (1994). Interocularly unpaired zones escape local binocular matching. Vision Research, 34, 1875-1881.

Shimono, K., Ono, H., SAIDA, S., \& MaPp, A. P. (1998). Methodological caveats for monitoring binocular eye position with nonius stimuli. Vision Research, 38, 591-600.

Usher, M., \& Donnelly, N. (1998). Visual synchrony affects binding segmentation in perception. Nature, 394, 179-182.

van Ee, R., Banks, M. S., \& Backus, B. T. (1999a). An analysis of binocular slant contrast. Perception, 28, 1121-1145.

van Ee, R., Banks, M. S., \& Backus, B. T. (1999b). Perceived visual direction near an occluder. Vision Research, 39, 4085-4097.

Wheatstone, C. (1838). Contributions to the physiology of vision: Part the first. On some remarkable and hitherto unobserved phenomena of binocular vision. Philosophical Transactions of the Royal Society of London, 128, 371-394.

Wolfe, J. M. (1986). Stereopsis and binocular rivalry. Psychological Review, 93, 269-282.

\section{NOTES}

1. Effects of binocular stimuli on monocular elements have also been found by Rogers and Bradshaw (1999) and Shimono, Ono, Saida, and Mapp (1998).

2. A degenrate case would be that the line is lying on the horizontal plane. In such a case, the two points $\mathrm{P}_{0}$ and $\mathrm{P}_{1}$ of Figures 4 and 5 would project to a single point on the retina, and the stimulus would be indistinguishable from the projection of a single point. However, this ambiguity is never present in our display, since the two extremes are always separated vertically.

3. Since the trend of the data is similar for all the subjects, the psychometric function of only 1 subject is presented. 


\section{APPENDIX}

Let us assume that a point $\mathrm{P}(x, z)$ which projects a horizontal coordinate $u$ on the image plane is seen only by the right eye. Let us also assume that both eyes are fixating a point $\mathrm{F}$ that is the origin of a Cartesian coordinate system. Hering's (1879/1942) law about perceived visual direction states that the perceived visual direction of the point $\mathrm{P}$ by the cyclopean eye is the angle $\alpha_{\mathrm{r}}$ between the visual axis of the right eye and the line connecting the nodal point of the right eye and the point $\mathrm{P}$ (see Figure A1). The tangent of the angle $\alpha_{\mathrm{r}}$ is

$$
t_{\mathrm{r}}=\tan \left(\alpha_{\mathrm{r}}\right)=\frac{u d}{d^{2}-u e+e^{2}},
$$

where $d$ is the distance from the eyes to the image plane and $e$ is half the distance between the nodal points of the two eyes. If $z$ is the depth coordinate of the point $\mathrm{P}$ and $D$ is the distance of the cyclopean eye from the image plane, then the horizontal position $x$ of $\mathrm{P}$ can be calculated by the following equation:

$$
x=(D+z) \tan \left(\alpha_{\mathrm{r}}\right),
$$

where $D$ is the sum of $d$ and $\Delta$ (see Figure A1). By substituting Equation A1 in Equation A2, we obtain

$$
x=\frac{u(D+z)}{D-u t_{f}},
$$

where $t_{f}$ is the tangent of half the vergence angle $\alpha_{f}$ and is equal to $e / d$.

Let us now consider a further point $\mathrm{P}^{\prime}(x+\Delta x, z+\Delta z)$, which projects on the image plane at the horizontal coordinate $u+\Delta u$. By calculating the difference between the $x$ coordinates of the points $\mathrm{P}^{\prime}$ and $\mathrm{P}$, we obtain the following equation:

$$
\Delta x=\frac{2 D(D+z)}{\left(D-u t_{f}\right)^{2}} \Delta_{u}+\frac{2 u}{\left(D-u t_{f}\right)} \Delta z,
$$

which can be simply rewritten as

$$
\Delta x=k_{u}(e, d, u, z) \Delta u+k_{z}(e, d, u) \Delta_{z} .
$$

This equation represents the fact that if two points are located at different distances $(\Delta z<>0)$ and they project to the same horizontal coordinate on the image plane $(\Delta u=0)$, their horizontal location is different. It is important to note that this difference $(\Delta x)$ has, in general, opposite signs in the two visual fields.

Let us assume that a relative depth $\Delta z$ is perceived between the monocular elements $\mathrm{P}$ and $\mathrm{P}^{\prime}$. If this is the case, the second term of the right hand of Equation A5 should be different from zero. If a psychometric function has as an independent variable $\Delta u$ and as a dependent variable the probability of, say, perceiving $\Delta x>$ 0 , the term $k_{u}$ represents the slope of the psychometric function, and the product $k_{z}(e, d, u) \Delta z$ the bias-that is, the shift of the psychometric function from a mean of zero. If we collect the psychometric function on the

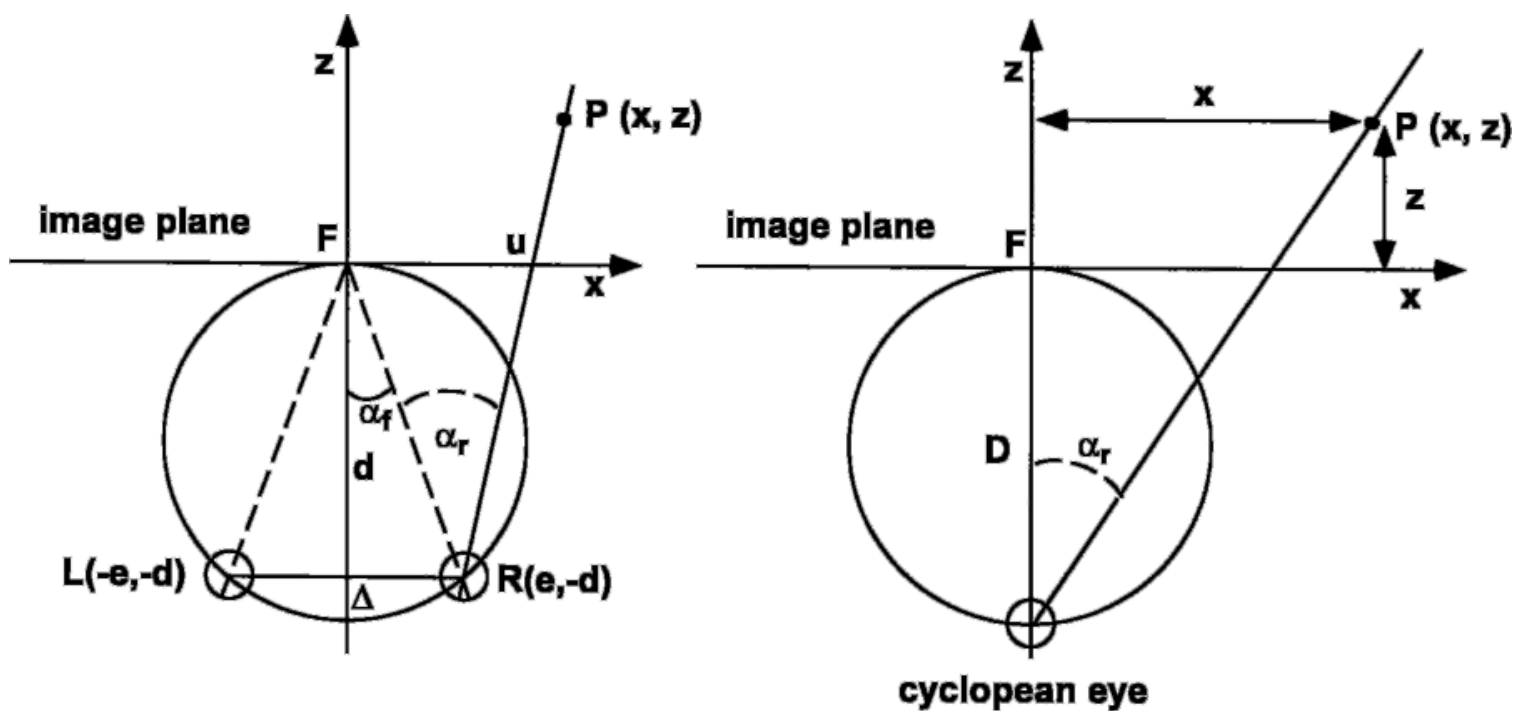

Figure A1. Sketch of the prediction of perceived visual direction according to the Hering (1879/1942) law. The left panel shows a viewing situation in which an element $P$ is seen only by the right eye. The right panel represents the visual direction of the element $P$ that would be perceived by the cyclopean eye. 


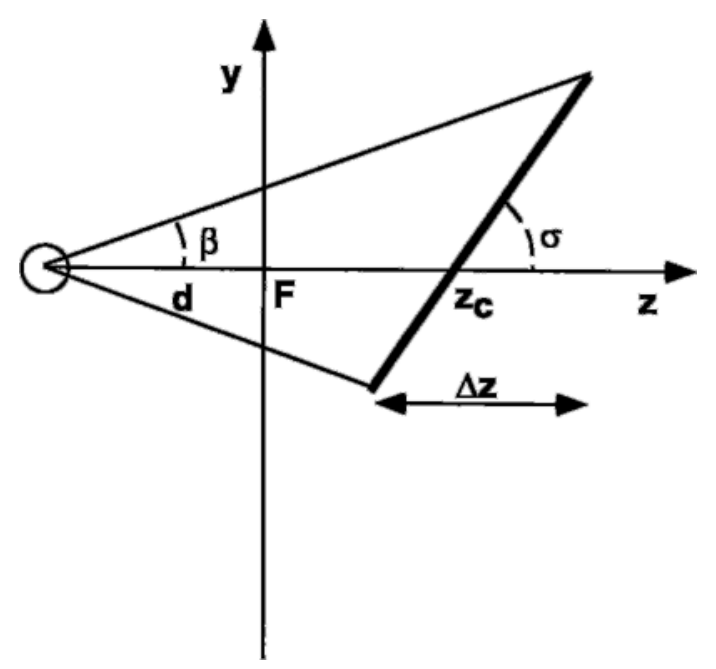

Figure A2. Viewing geometry of a slanted planar surface in the $y-z$ plane.

left and right visual fields at the same distance $|u|$ from the fixation point, the difference between the shifts of the psychometric functions is represented by

$$
k_{z}(e, d,|u|) \Delta z-k_{z}(e, d,-|u|) \Delta z=\frac{4|u| d}{D^{2}-\frac{u^{2} t_{f}^{2}}{}} \Delta z .
$$

Therefore, the difference between the $50 \%$ points of the psychometric functions computed for the left and right visual fields is proportional to the perceived relative depth.

If two elements that project a vertical separation $\beta$ on the image plane are located on a surface slanted with slant $\sigma$ (see Figure A2), it can be shown that the depth separation between these elements is

$$
\Delta z=\frac{2\left(d+z_{c}\right)}{\left(\frac{t_{\sigma}}{t_{\beta}}-\frac{t_{\beta}}{t_{\sigma}}\right)},
$$

where $t_{\beta}$ and $t_{\sigma}$ are the tangents of the angles $\beta$ and $\sigma$, respectively. Equation A7 reveals that the depth separation of two elements that project on the image plane with the same vertical separation $\beta$ is an increasing function of the tangent of the slant $\sigma$ of the surface on which they are located. 